\title{
Visual Information Retrieval for Videos Based on Feature Extraction using Machine Learning Techniques
}

\author{
V.Mohana Maniganda Babu, S.Sasireka, E.Anitha
}

\begin{abstract}
Information retrieval is one of the important areas of research with highest scope for data mining combined with machine learning. The proposed research focus on visual information retrieval by applying machine learning techniques. The usage of multimedia data such as text, images, videos are abundantly increasing day by day in this smart era. Also the need for information classification and retrieval are getting exponential demands to fulfill the research and end user requirements. The tech giants are conducting their researches to develop efficient retrieval systems for videos. Video retrieval is considered to be the toughest and challenging research in the recent times. Due to large storage space, lengthy play time, multiple sequence of frames, spatial temporal challenges, lack of visual relevancy, less hardware and processing support. The proposed visual information retrieval has got higher scope of research with the above listed problems.
\end{abstract}

Index Terms: Bag of features (Bof), histograms, Support Vector Machines, key point locations-Means algorithm.

\section{INTRODUCTION}

Gathering examination is an extensively associated methodology in data mining. Nevertheless, most of the current bundling in figures are not capable in overseeing discretionarily formed transport data of to an incredible degree colossal and high-dimensional datasets. On the other hand, estimations based cluster endorsement techniques realize high computational expense in gathering examination which continues packing of figures from being effectively used before long. Observation methodologies have been brought into group examination. In any case, most recognition strategies used in gathering examination are generally used as contraptions for information rendering, instead of for investigating how data lead changes with the assortments of the parameters of the counts. In addition, the incorrectness of portrayal limits its convenience in separating gathering information of data.

Video contains tremendous proportions of data which ought to be made and compacted in a capable manner (e.g., one hundred extensive stretches of video contains around

Revised Manuscript Received on July 08, 2019.

V.Mohana Maniganda Babu, School of IT \& Science, DR.G.R. Damodaran College of Science, Coimbatore.

S.Sasireka, Department of Computer Science and Engineering, Bannari Amman Institute ofTechnology, Sathyamnagalam, Erode.

E.Anitha, Department of Computer Science and Engineering,Bannari Amman Institute of Technology, Sathyamnagalam, Erode. 10million edges requiring around 7:5TeraBytes of data). Progressing work in mechanized video recuperation has stressed on a different leveled depiction of video for straightforwardness of perception, addressing, scrutinizing, and requesting. In the midst of the parsing method, video cuts are parceled into scenes. Scenes are moreover divided into shots which are each addressed similarly as two or three key edges. A scene which speaks to the most elevated amount of pecking order comprises of a gathering of shots that speak to a dynamic significance, for example, a shoreline scene, an exchange in a café, a wedding, and so on. A shot is a progressive arrangement of casings speaking to a delayed activity in existence. Along these lines, in the situation of an eatery exchange between Mr. X and Ms. Y, a shot may comprise of the arrangement of casings focusing on Ms. $\mathrm{Y}$ as she addresses Mr. X. A shot for the most part comprises of different edges, a considerable lot of which are fundamentally the same as in substance. Semantic substance of the shot is caught by speaking to each shot with an insignificant arrangement of key casings. Programmed plans for shot recognition and resulting key edge extraction have been accounted for in the writing.

However, a video clip may contain a number of shots. For example, report upto 30shots in a 15 minute clip of Terminator 2 and a 30minute clip of sitcom "Frasier". Assuming an average of key frames per shot, close to 1; 0 key frames would be required to represent these video clips. In a digital library with over 10hours of digitized video, about 10;0 key frames may be extracted. Indexing and clustering of these key frames would then allow the users to jump across video clips to location of their interest. Our goal is to develop a scheme for automatic classification of key frames.

Intriguing examples are portrayed and found utilizing information mining . This assignment is particularly testing when the information comprise of video groupings (which may likewise have sound substance), in view of the need to break down gigantic volumes of multidimensional information. The wealth of the area [3] suggests that a wide range of methodologies can be taken and various apparatuses and systems can be utilized, as can be found in the parts of this book. They manage grouping and arrangement, prompts and characters, division and rundown, insights and semantics. In the creators' own (sometimes abbreviated) words, the parts manage video perusing utilizing various synchronized perspectives; the 
physical setting as a video mining crude; worldly video limits; video synopsis utilizing action and sound descriptors; content investigation utilizing multimodal data; video OCR; video arrangement utilizing semantics and semiotics; the semantics of media; factual systems for video examination and seeking; mining of measurable transient structures in video; and pseudo-importance input for mixed media recovery.

Digital video is a rich medium compared to text material. It is usually accompanied by other information sources such as speech, music and closed captions. Therefore, it is important to fuse this heterogeneous information intelligently to fulfill the users' search queries. Conventionally, the data is often indexed and retrieved by directly matching homogeneous types of data. Multimedia data, however, also contains important information related to the interaction between heterogeneous types of data, such as video and sound, a fact confirmed through human experience. We often observe that a scene may not evoke the same response of horror or sympathy, if the accompanying sound is muted. Conventional methods fail to utilize these relationships since heterogeneous data types cannot be compared directly. The challenge is to develop sophisticated techniques that fully utilize the rich source of information contained in multimedia data.

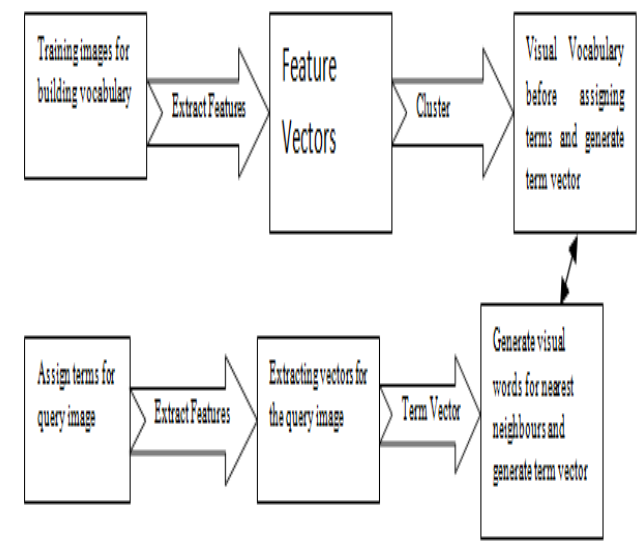

Fig1.Building vocabulary and generate vectors

\section{K-Means Algorithm ApProach}

After the metric space definition, in past module k-implies calculation is executed on preprocessed information for introductory bunch strategy. They begin with a parcel calculation with groups, with essentially littler than the aggregate number. As indicated by the separation metric, we take the most distant couples and decide interims. Primary objective is to abuse this property to aggregate associations (objects) to recognize client sessions (bunches) in a programmed mold.

[4] Depending on the data set, an ad hoc distance definition should be provided on the basis of a trial and error procedure. Let us assume for simplicity that $X=R n$, where is the sampling space dimension. Represents the component of sample is $\mathrm{xi}$, the sample distance

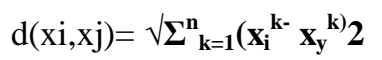

is the classical Euclidean metric, and the distance between two clusters, is defined as

$$
\mathrm{d}\left(\mathrm{C}_{\mathrm{i}, \mathrm{Cj}}\right)=\min _{\mathrm{xeR}(\mathrm{Ci}), \mathrm{yeR}(\mathrm{Cj})} \mathrm{d}(\mathrm{x}, \mathrm{y}) \ldots(\mathrm{(})
$$

where $\mathrm{R}(\mathrm{C})$ is a set of selected points representing the whole cluster.

K-Means calculation is utilized when the last number of bunches is known. The technique begins with an underlying design including groups, chose by a few criteria. The last bunch definition is gotten through an iterative system. The bunch is spoken to by a subset of tests when estimating group to-group separate, where is the extent of the inspecting space, at technique startup, bunches are made, with bunch centroids chose by a given control in the estimation space, e.g., to segment the space in equi-divided zones or haphazardly. Each sample is associated with the closest cluster, based on the distance between the samples and the centroids of each cluster.

\section{A. Algorithm Implementation}

There are $\mathrm{N}$ test include vectors $\mathrm{x} 1, \mathrm{x} 2, \ldots, \mathrm{xn}$ all from a similar class, and we realize that they fall into $\mathrm{k}$ minimized bunches, $\mathrm{k}<\mathrm{n}$. Give mi a chance to be the mean of the vectors in bunch I. On the off chance that the bunches are all around isolated, we can utilize a base separation classifier to isolate them. That is, we can state that $\mathrm{x}$ is in group I if $\| \mathrm{x}$ $\mathrm{mi} \|$ is the base of all the $\mathrm{k}$ separations. This recommends the accompanying technique for finding the $\mathrm{k}$ implies:

- Make introductory estimates for the methods $\mathrm{m} 1, \mathrm{~m} 2$, mk Repeat until there are no adjustments in any mean and the intends are assessed

- Use the assessed intends to arrange the examples into groups

- For I from 1 to $\mathrm{k}$, then replace the mean

- Replace mi with the mean of the majority of the examples for group I

- End for

- End until

- B.Scale-space extrema detection

Key points are the intrigue focuses.

Difference of Gaussians (DoG) for DoG image

$\mathrm{D}(\mathrm{x}, \mathrm{y}, \sigma)$

$$
\mathrm{D}(\mathrm{x}, \mathrm{y}, \sigma)=\mathrm{L}(\mathrm{x}, \mathrm{y}, \mathrm{ki} \sigma)-\mathrm{L}(\mathrm{x}, \mathrm{y}, \mathrm{kj} \sigma)
$$

$\mathrm{L}(\mathrm{x}, \mathrm{y}, \mathrm{k} \sigma)$ is the convolution of the original image $\mathrm{I}(\mathrm{x}, \mathrm{y})$ with the Gaussian $\mathrm{G}(\mathrm{x}, \mathrm{y}, \mathrm{k} \sigma)$ is the blur a scale $\mathrm{k} \sigma$, i.e.,

$\mathrm{L}(\mathrm{x}, \mathrm{y}, \mathrm{k} \sigma)=\mathrm{G}(\mathrm{x}, \mathrm{y}, \mathrm{k} \sigma) * \mathrm{I}(\mathrm{x}, \mathrm{y})$

Consequently a DoG picture amongst scales and is only the distinction of the Gaussian-obscured pictures at scales and. Scale space extrema location in the SIFT calculation, the picture is first convolved with Gaussian-obscures at various scales. The convolved pictures are gathered by octave (an octave compares to multiplying the estimation of), and the estimation of is chosen with the goal that we get a settled number of convolved pictures per octave. At that point the Difference-of-Gaussian pictures are taken from contiguous Gaussian-obscured pictures per octave. 


\section{SUPPORT VECTOR MACHINE}

Machine leaning manages the leaning models by using, the Support vector machines that examine the information and designs are perceived with appropriate calculations which utilize the method of grouping the examination. The test cases are prepared by using the two classification apart from assets. Non-probabilistic paired direct classifier is formed by SVM model constructed assigns new cases to one class by the prepared calculations. Different classifications are separated by unmistakable hole under the certain circumstances. Same space is allocated for new illustrations. Non-direct characterization can be played with SVM by utilizing the play trap. These mapping contributes an element spaces with high dimension.

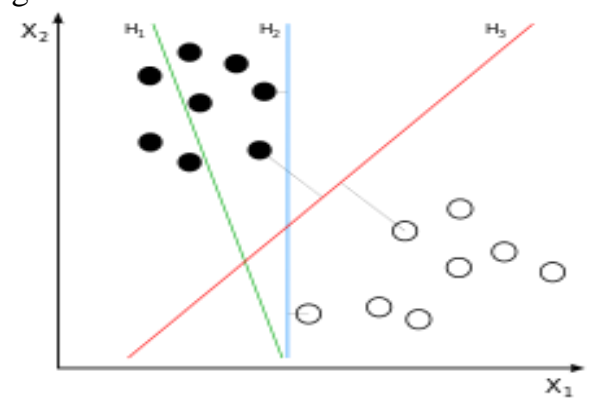

Training image features can be detected wit image scale, noise and illumination. These points lie object edges which is a high-contrast regions. The relative positions should not change in the original scene. Articulated or flexible objects along with the features are easily locatedWhere the $n$ points are constituted by data $\mathrm{D}$ of training set.

$$
\mathrm{D}=\{(\mathrm{xi}, \mathrm{yi}) \mid \mathrm{xi} \in \mathrm{Rp}, \text { yi } \in\{-1,1\}\} \mathrm{n} \mathrm{i}=1
$$

yi indicates the class of xi.

$\mathrm{p}$ - Vector is $\mathrm{xi}$.

$\mathrm{yi}=1$ is divided to find the maximum-margin hyper plane

The value of Support Vector Machine is positive if the videos from the database are matched and retrievd.It stays negative, if the values are not matched.

Support vectors are

w. $x-b=0$

The parameter $\frac{b}{\|\mathbf{w}\|}$ is the offset.

Equation of hyper planes are

$\mathrm{w} \cdot \mathrm{x}-\mathrm{b}=1 \quad$ and

w. $x-b=-1$

Minimizing the distance between the two hyper planes, w.xi-b $>=1$ for $x i$ of the first class

or

w. $x i-b<=-1$ for $x i$ of the second class

Or

Yi(w.xib?) $>=1$ for all $1<=\mathrm{i}<=\mathrm{n}$,

- A.Primal form

Training vectors with linear combination

$\mathrm{W}=\boldsymbol{\Sigma}_{\mathbf{i}=\mathbf{1}}{ }^{\mathbf{n}} \boldsymbol{\alpha}_{\mathbf{i}} \mathbf{y}_{\mathbf{i}} \mathbf{X}_{\mathbf{i}}$

Support vectors can be,

w.wi $-\mathrm{b}=1 / \mathrm{yi}=\mathrm{yi} \Leftrightarrow \mathrm{b}=\mathrm{w}$

Unbiased estimator,

$\mathrm{B}=1 / \mathrm{N}_{\mathrm{SV}} \boldsymbol{\Sigma}^{\mathrm{NSV}}{ }_{\mathbf{i}=\mathbf{1}}\left(\mathbf{w} \cdot \mathbf{X}_{\mathrm{i}}-\mathbf{y}_{\mathbf{i}}\right)$.

\section{EXPERIMENTAL RESULTS AND DISCUSSION}

\section{A. Datastore}

The database for the proposed system large number of video clips downloaded from the internet. The total play length time of video clip data is approximately one hundred and sixty hrs which includes all the contents like news, sports, movie, cartoon, teleplay and natural scene etc. The experiments were performed on Xeon E5410 2.33GHz, 2G memory. The algorithms implemented are used in comparison table for comparing the results. The experiments were set to the performance comparison of similarity search.

The database for the proposed framework huge number of video cuts downloaded from the web. The all out play length time of video cut information is roughly one hundred and sixty hrs which incorporates every one of the substance like news, sports, film, animation, teleplay and characteristic scene and so forth. The tests were performed on Xeon E5410 $2.33 \mathrm{GHz}, 2 \mathrm{G}$ memory. The calculations executed are utilized in correlation table for looking at the outcomes. The tests were set to the presentation examination of closeness seek. The framework picks the most provoking 40 questions to inquiry the recordings from a video database. Information Queries are picked and coordinated from the recordings accessible in the video database. Every year google looks at billions of inquiries that individuals around the globe have composed into google inquiry to find the spared EVVE video Archives. To gather the video files information from EVVE site and pick the most unpredictable to questions to look video. The quantity of downloaded recordings for each question is up excessively 100. Removed in excess of 50 recordings are from the video database files.

\section{B. Comparative Study}

A few recordings have been performed utilizing the tangle lab to assess the effect of various kinds of comparative recordings. Bolster Vector Machine (SVM) is performed on the comparable recordings in which the right and fitting position and edges are recovered from the comparable recordings.

Programmed Extraction of Similar Videos utilizing a video inquiry cut dependent on Support Vector Machine contributes in a few recordings of a few demonstrating and comparable video extraction, examine the region process. By looking at the two strategies for Support Vector Machine and VISCOM model the exactness rate and time are contrasted with recover the comparative video from the database.

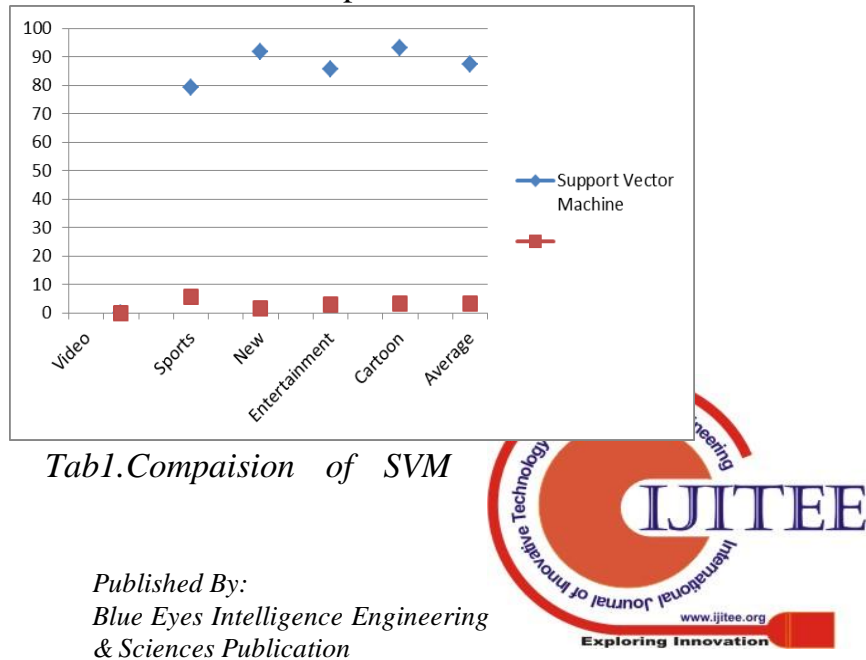




\section{and $K$ Means}

The Similar video extraction process is naturally performed and is utilized for extra reason (VISCOM) recordings model to play out the extraction of comparable procedure.

Exactness rate and time of an each procedure in both the techniques are decreased when contrasted with the past strategy for an occasion.

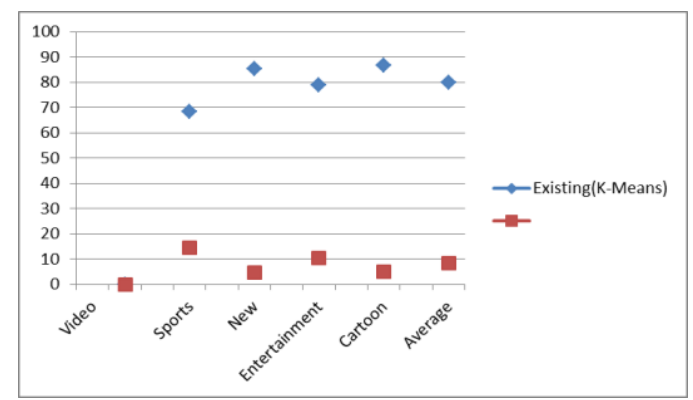

Fig 1.Accuracy of K-Means

\section{EXPERIMENTAL RESULTS}

The algorithm (1) is the method and the algorithm (2)

\begin{tabular}{|c|c|c|c|c|}
\hline \multirow[b]{2}{*}{ Video } & \multicolumn{2}{|l|}{$\begin{array}{l}\text { Support } \\
\text { Machine }\end{array}$} & \multicolumn{2}{|c|}{$\begin{array}{l}\text { Existing(K-Mea } \\
\text { ns) }\end{array}$} \\
\hline & Accuracy & Time & $\begin{array}{l}\text { Accura } \\
\text { cy }\end{array}$ & me \\
\hline $\begin{array}{ll} & \text { Sport } \\
\text { s } & \\
\end{array}$ & 79.32 & 5.44 & 68.50 & $26^{14 .}$ \\
\hline New & 91.65 & 1.45 & 85.43 & $2^{4.4}$ \\
\hline $\begin{array}{ll} & \text { other } \\
\mathrm{s} & \\
\end{array}$ & 85.76 & 2.82 & 78.89 & 28 \\
\hline $\begin{array}{l}\text { Carto } \\
\text { on }\end{array}$ & 93.13 & 3.32 & 86.74 & $0^{4.8}$ \\
\hline $\begin{array}{l}\text { Aver } \\
\text { age }\end{array}$ & 87.47 & 3.26 & 79.89 & $\begin{array}{ll} & 8.4 \\
7 & \end{array}$ \\
\hline
\end{tabular}

stands for the approach. The precision rate goes down when the recall rate rises. The proposed method holds steady precision at 1 while the others drop significantly when the recall rate rises. The algorithm (1) is slightly better than algorithm (2) in precision rate when the recall rate is smaller than 0.60 . The proposed algorithm can hold precision rate at 1 when the recall rate reaches 0.75 . The others have the best precision rate at 0.7 when the recall rate is 0.75 . When recall rate is approaching to 1 , the precision rate of the proposed method decreased a little at 0.9 .

The other's precision rate are below 0.6 when their recall rate are higher than 0.8. This is because the proposed search approach is according to video components based on the statistics of STD. The similarity search is directed to the corresponding CIT and the dissimilar video can be eliminated effectively by index clustering. The representative video shot images of comparison VSS.

The proposed method can find more similar video clips with similar shots compared with the algorithm based on support vector machine. Support Vector Machine algorithm can perform twenty times faster than the other algorithms. The reason is that the proposed search is carried out in a bounded scope based on video components of relevant similar video and redundant search of dissimilar video can be avoided.

The extensive opportunity suppositions are rarely wrong, to vanquish this honest Bayes classifier is used that has a couple of properties to make it before long. In particular, the decoupling of the class unexpected segment scatterings infers that each spread can be unreservedly evaluated as a one dimensional course. This subsequently facilitates issues originating from the scourge of dimensionality, for instance, the prerequisite for instructive records that scale exponentially with the amount of features. Like each probabilistic classifier under the MAP decision control, it gets in contact at the correct request as long as the correct class is more conceivable than some different class; therefore class probabilities don't should be assessed extraordinarily well. In a manner of speaking, the general classifier is adequately solid to neglect certifiable needs in its basic straightforward probability illustrate. Various clarifications behind the watched achievement of the guiltless Bayes classifier are analyzed in the composing referred to beneath. Database Setup

The database in the trials was comprised of one thousand video cuts downloaded from web. The absolute length of video information is around one hundred and sixty hrs, including different substance, for example, news, sports, motion picture, animation, teleplay and regular scene and so forth. The investigations were performed on Xeon E5410 $2.33 \mathrm{GHz}, 2 \mathrm{G}$ memory. The calculations in were actualized for correlation.

\section{A. Examination}

The investigations were set to the presentation examination of closeness look. The consequences of review accuracy bends are appeared. The calculation (1) is the technique and the calculation (2) represents the methodology [9]. The exactness rate goes down when the review rate rises. The proposed strategy holds unfaltering exactness at 1 while the others drop essentially when the review rate rises. The calculation (1) is somewhat superior to calculation (2) in exactness rate when the review rate is littler than 0.60 .

In addition, the hunt time of different calculations becomes quicker than the proposed methodology. This is on the grounds that the proposed hunt is guided into the comparing database CIT whose volume isn't extent to that of video database. As indicated by test results, the proposed technique can enormously improve the productivity of video comparability seek in huge database.

The proposed calculation can hold exactness rate at 1 hen the review rate achieves 0.75 . The others have the best accuracy rate at 0.7 when the review rate is 0.75 . At the point when review rate is drawing closer to 1 , the exactness rate of the proposed strategy diminished a little at 0.9. The others' exactness rate are underneath 0.6 when their review rate are higher 
than 0.8 . This is on the grounds that the proposed inquiry approach is as indicated by video parts dependent on the measurements of STD. The similitude seek is coordinated to the relating CIT and the disparate video can be wiped out successfully by record bunching. The agent video shot

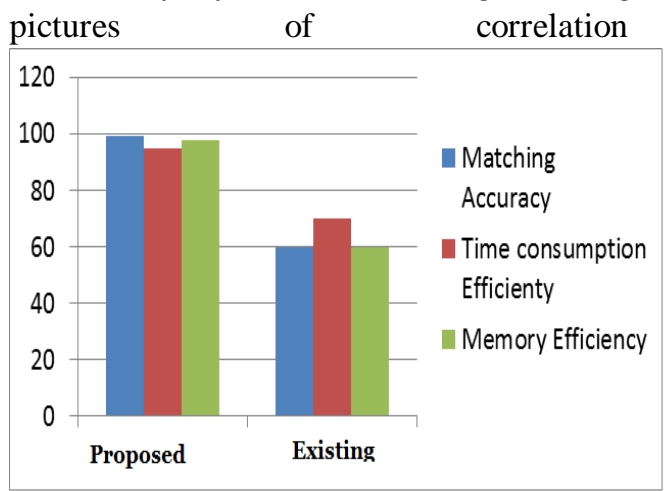

Fig 2. Accuracy Chart

By giving question video, the proposed technique can discover progressively comparative video cuts with comparable shots contrasted and the calculation (1). Consequently, the better VSS execution can be acquired by proposed technique with fulfilling review and accuracy rate as it is appeared in Fig.4.1. The consequences of normal pursuit time versus the quantity of video are appeared in Fig.4.2.

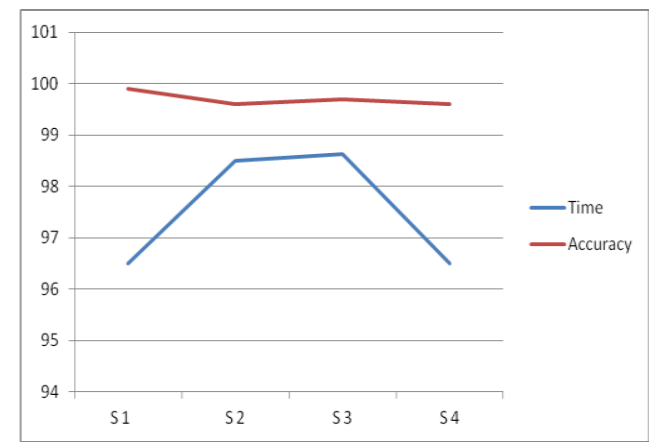

Fig 3. Accuracy Chart

The comparison is based on the average generation time of VST in proposed VSS system. The real query of VSS system is extremely fast because it just shows the content of VST. With the growing number of video clips, the search time increases.

\begin{tabular}{|l|r|r|r|r|}
\hline $\begin{array}{l}\text { Comparat } \\
\text { ive } \\
\text { Analysis }\end{array}$ & \multicolumn{1}{|c|}{$\begin{array}{r}\text { S } \\
\text { Time }\end{array}$} & \multicolumn{2}{|c|}{ S 3 } & \multicolumn{2}{|c|}{ S } \\
\hline & 6.5 & 8.5 & 98 & 9 \\
\hline Accuracy & 9 & 9 & 99 & 6.5 \\
& 9.9 & 9.6 & .7 & 9.6 \\
\hline
\end{tabular}

\section{CONCLUSION}

The proposed algorithm performs about twenty times faster than the other algorithms. The reason is that the proposed search is carried out in a bounded scope based on video components of relevant similar video and redundant search of dissimilar video can be avoided. Besides, the search time of other algorithms grows faster than the proposed approach. This is because the proposed search is guided into the corresponding database CIT whose volume is not proportion to that of video database. According to experimental results, the proposed method can greatly improve the efficiency of video similarity search in large database.

Our proposed research focus on providing an efficient solution to various challenges such as

- Increasing visual relevancy during retrieval process.

- Retrieval of videos based on spatio temporal relationship.

- Designing efficient methodology to increase the processing speed with the available resources.

- Develop a retrieval system for processing lengthy video sequence.

- To retrieve exact videos from a large video database of any size.

By applying machine learning techniques, learning the visual characteristics of videos in large video database and applying visual information retrieval technique to retrieve the exact matching videos is proposed in this research. Efficiency of the proposed research can be tested by conducting various experiments on the following parameters such as retrieval time, retrieval accuracy and visual similarity of the input query and retrieval results.

We intend to additionally examine the impact of speaking to recordings by different highlights, for example, ordinal mark and furthermore to present fusing spatial data, consolidating Bof display with worldwide histogram highlights and extension of questions. Moreover, the weight of each factor for measuring video similarity might be adjusted by user feedback to embody the degree of similarity more completely and systematically. Video Compression and de-noising can be added in future to the current system. The System can include the video condensation approaches to reduce the size of the video file.

Relevance feedback can be added in future is due to its interaction with the user provides the ability to solve the video retrieval in low-level features and high-level semantic mapping between the problem has been widely studied and applied in the SVM algorithm, feedback classification and retrieval algorithms are the fastest-growing part, there are a lot of ways branch. However, annual annotation for the number of images and video library of the great differences between the proportions of the core issue still cannot be a good solution

- Effect of representing the videos by other features such as 3D, environmental Changes.

- The weight of each factor for measuring video similarity might be adjusted by user 
feedback to embody the degree of similarity more completely and systematically.

- Video Compression and de-noising can be added in future to the current system.

\section{REFERENCES}

1. Dinakaran.D, J. Annapurna, Ch. AswaniKumar,'Interactive Image Retrieval Using Text and Image Content" Cybernetics and information technologies, vol. 10, no. 3 pp.20-30,2010

2. Chin-Chen Chang and Tzu-Chuen Lu, "A Color-Based Image Retrieval Method Using Color Distribution and Common Bitmap" Springer, pp. 56-71, 2005

3. Kekre H.B, Sudeep D. Thepade, TanujaK. Sarode and Shrikant P. Sanas, "Image retrieval using texture features extracted using LBG, KPE, KFCG, KMCG, KEVR with assorted Color spaces" International Journal of Advances in Engineering \& Technology, Vol. 2, Issue 1, pp. 520-531,2012

4. Vivek Jain, Neha Sahu "A Survey: On Content Based Image Retrieval" International Journal of Engineering Research and Applications (IJERA) ISSN: 2248-9622, Vol. 3, Issue 4, Jul-Aug 2013, pp.1166-1169

5. V.MohanaManigandaBabu, Dr.T.Santha, "Efficient Brightness Adaptive Deep-Sea Image Stitching using Biorthogonal Multi-Wavelet Transform and Harris Algorithm", IEEE International Conference on Intelligent Computing and Control (I2C2 17),ISBN: 978-1-4673-9916-6,23-24th June 2017.

6. V.MohanaManigandaBabu, Dr.T.Santha, "Efficient Brightness Adaptive Deep-Sea Image Stitching using Biorthogonal Multi-Wavelet Transform and Harris Algorithm", IEEE International Conference on Intelligent Computing and Control (I2C2 17), ISBN: 978-1-5386-0374-1,23-24th June 2017.

7. Sakthi Sivkumar.V, "Organic On-Field Research Combined with Information Technology for a Fast Uptake, Sustainable and Profitable Future Using Both Macro and Micro Analysis of The System as a Whole", (ORGATROP 2017) - International Conference on Organic Agriculture in the Tropics: State-of-the-Art, Challenges and Opportunities, Yogyakarta, Indonesia, August 20 - 24, 2017.

8. Sakthi Sivakumar. V, "Applying both modern and ancient Management principles and planning to optimize the Organic sector from field level to increase the Sustainability and Profitability" (OWC - 2017), Organic World Congress, Greater Noida, India, Nov 09 -11, 2017.

9. M Abhayadev, Dr.T Santha, "Object Boundary Identification using Enhanced High Pass Frequency Filtering Algorithm and Morphological Erosion Structuring Element", Journal of Scientific \& Industrial Research (SCIE),Vol. 76, pp.620-625, October 2017

\section{AUTHORS PROFILE}

V.Mohana Maniganda Babu, School of IT \& Science, DR.G.R Damodaran College of Science, Coimbatore..

S.Sasireka, Department of Computer Science and Engineering, Bannari Amman Institute ofTechnology, Sathyamnagalam, Erode.

E.Anitha, Department of Computer Science and Engineering,Bannari Amman Institute of Technology, Sathyamnagalam, Erode. 